\title{
Indicadores de desempeño de procesos médicos con alineamiento estratégico en la atención al paciente
}

\author{
Performance indicators of medical processes with strategic alignment in patient care \\ Martha K. Torres-Salgado* \\ División de Investigación, Facultad de Contaduría y Administración, Universidad Nacional Autónoma de México, Ciudad de México, México
}

\begin{abstract}
Resumen
Los indicadores de desempeño (también conocidos como KPI [Key Performance Indicator]) de los procesos son de relevancia, de prioridad y se justifica su estudio, porque posibilitan medir para poder evaluar y mejorar la atención médica. El objetivo es dar respuesta al por qué son importantes los KPI en los procesos y cómo definirlos de acuerdo con su aplicación. Se refiere a un estudio analítico, conceptual y con razonamiento deductivo, de alcance explicativo para su correlación de KPI estratégicos a los procesos, de acuerdo con la importancia de las actividades en los servicios médicos. El resultado lleva a comprender la propuesta de modelo de los KPI para procesos con niveles de alineamiento estratégico. Finalmente, se presenta el modelo de KPI para procesos con niveles de alineamiento estratégico, que visualiza de manera integral y que coadyuva en su aplicación a mejorar la calidad en el servicio médico al paciente en el proceso.
\end{abstract}

Palabras clave: Planeación estratégica. Indicadores. Procesos médicos.

\begin{abstract}
The key performance indicators (KPI) to the processes are of relevance, of priority and their study is justified, because they make it possible to measure in order to evaluate and improve medical care. Whose objective is to respond to why the KPI are important in the processes and how to define them according to their application. It refers to an analytical, conceptual and deductive reasoning study. Explanatory scope for its correlation of strategic KPI to processes, according to the importance of activities in medical services. The result leads to an understanding of the KPI model proposal for processes with strategic alignment levels. Finally, the KPI model for processes with levels of strategic alignment is presented, which it visualizes in an integral way and that contributes in its application to improve the quality in the medical service to the patient in the process.
\end{abstract}

Key words: Strategic planning. Indicators. Medical processes.

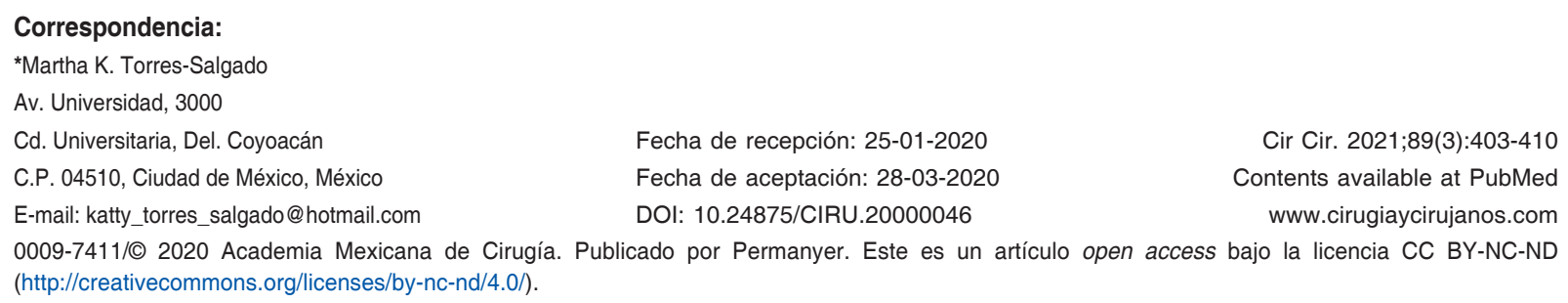

Fecha de recepción: 25-01-2020 Fecha de aceptación: 28-03-2020 C.P. 04510, Ciudad de México, México E-mail: katty_torres_salgado@ hotmail.com DOI: $10.24875 / C I R U .20000046$

Cir Cir. 2021;89(3):403-410 Contents available at PubMed www.cirugiaycirujanos.com 0009-7411/@ 2020 Academia Mexicana de Cirugía. Publicado por Permanyer. Este es un artículo open access bajo la licencia CC BY-NC-ND (http://creativecommons.org/licenses/by-nc-nd/4.0/). 


\section{Introducción}

La planeación estratégica es el proceso de establecer una visión institucional como sueño realizable de directivos, compartido por toda la comunidad organizativa, que permite centrar los recursos de la institución en los objetivos prioritarios que la impulsarán hacia su visión. Y es la parte de esclarecer la misión o finalidad fundamental de la institución, para proyectarla en una imagen de futuro, deseable, alcanzable y guiada por sus valores. Es un proceso que permite visualizar, de manera integrada, el futuro de las instituciones, la planeación estratégica, entendida como el proceso de conducir a una organización desde donde está y es en el presente, hasta donde deberá estar y ser en el futuro.

Las organizaciones son creadas en forma vertical sobre la base de la especialización para el cumplimiento de las diversas funciones que desarrollan. Es así que la responsabilidad por los resultados obtenidos se establece sobre la función y no sobre los procesos que se desarrollan en forma transversal a las unidades de la organización. La visión transversal sustentada en los procesos de la organización permite integrar los esfuerzos en torno a un objetivo común, comprender las dinámicas interacciones que se producen entre la empresa, los clientes, los proveedores y otros actores clave, y alinear el desempeño de la organización a la estrategia y los objetivos del negocio'1.

La evaluación parte del proceso de la planeación estratégica, es un instrumento básico para el logro de la misión y la visión organizativa, porque permite la mejora continua de cualquier organización. En cuanto a los indicadores de desempeño (KPI, Key Performance Indicator), por un lado la palabra «indicador» sirve para indicar y proviene del latín indicare, que significa mostrar o significar algo con indicios y señales; y por otro lado, «desempeño» es la capacidad de una organización para gestionar sus recursos y dar cumplimiento a los objetivos y metas establecidos ${ }^{2}$.

Ahora, en la relación de insumos versus productos proporcionan medidas útiles administrativas, siempre y cuando se establezca el indicador con el que habrá que medir esa relación ${ }^{3}$. Aunado a lo anterior, un indicador es un vehículo para medir valores en una escala de medición, derivados de una serie de hechos observados ${ }^{4}$.

Un indicador es un indicio, que deberá reflejar peculiaridades y nexos de los procesos que se originan en las actividades. También establece un soporte de información, habitualmente en expresión numérica, que representa una magnitud, de manera que a través de su análisis se permite la toma de decisiones sobre los parámetros asociados mediante las variables de control ${ }^{5}$.

\section{Análisis para la construcción de indicadores en la atención médica}

El análisis parte de la importancia de los indicadores en cuanto a su identificación, selección y formulación adecuada, que van a evaluar y tener el control del proceso; por ello, se requiere que los indicadores tengan ciertas características de:

- Representatividad, en relación a lo que se pretende medir.

- Sensibilidad, que es la flexibilidad de incorporar el cambio en la magnitud que representa.

- Rentabilidad, que es el beneficio que se obtiene al establecer, recopilar, calcular y analizar los datos del indicador.

- Fiabilidad, que es la confiabilidad de los datos obtenidos para obtener mediciones objetivas.

- Relatividad en el tiempo, es decir, que se pueda comparar en el tiempo para analizar su evolución y tendencias de futuro ${ }^{5}$.

En consecuencia, se puede apreciar entonces que se tiene necesidad de destacar la importancia de criterios entre la planeación estratégica y los procesos para establecer los indicadores.

Hay diversas propuestas para la construcción de indicadores. A continuación se presentan dos, la primera de ellas con cinco pasos 5 :

1) Reflexionar sobre el objetivo del proceso en la generación de un bien o servicio.

2) Determinar el tipo de resultados a obtener y las magnitudes a medir en la variabilidad del proceso.

3) Determinar y formular los indicadores que se van a emplear e implementar, representativos de lo que se pretende medir.

4) Establecer resultados esperados.

5) Formalizar los indicadores con los resultados $y$ los objetivos que se desea alcanzar.

La segunda propuesta es para establecer la construcción de indicadores y los tableros de indicadores en seis etapas ${ }^{6}$ :

1) Identificación de indicadores. Para la construcción de la arquitectura de indicadores en procesos prioritarios de actividades concretas se basa 
en los procesos, subprocesos y etapas en la obtención de los mapas de procesos.

2) Elección de actividades de prioridad en los mapeos. Se elige la actividad de mayor importancia dentro del proceso, no necesariamente en los insumos o resultados del proceso para actividades de prioridad o concretas, lo que permite de manera preventiva garantizar el resultado esperado del bien o servicio. Las actividades de prioridad se identifican claramente en la cadena de valor como actividades únicas y distintivas que entregan de forma satisfactoria el bien o servicio, o de lo contrario estas actividades se convierten en un cuello de botella que impactaría en la entrega de un bien o servicio insatisfactorio y en conflictos entre las personas ${ }^{7}$.

3) Definición de indicadores. Definir los KPI que arrojen mediciones útiles, precisas, correctas e importantes es garantía de llevar una buena estrategia y tomar decisiones relevantes. EI KPI ofrece la visualización de las actividades para anticiparse a los resultados y poder tomar las decisiones desde cualquier área funcional en que se ubique la actividad.

4) Implementación de indicadores y validación. Es la formalización a través del documento que permite estandarizar los principales elementos que integran el indicador, es el diseño de ficha de indicadores tipo, que permite dar seguimiento a través del monitoreo por el actor o dueño responsable de reportar los avances. Además, sirve para evidenciar y validar si el control establecido es el idóneo y funciona para recopilar la información estadística y consolidar su ajuste e implementación, e incorporarlo como parte del tablero para el grupo operativo, los mandos medios 0 bien la alta dirección.

5) Perspectivas de indicadores. La categorización permitirá agruparlos de acuerdo con cierto criterio para visualizar el avance de los objetivos de cada grupo de indicadores de manera puntual:

- Rentabilidad (productividad). Se refiere a recuperar la inversión y obtener beneficios financieros en costo/beneficio. Para los hospitales es común utilizar la productividad que se refiere a resultados en los servicios de atención médica.

- Actividades. Eslabón prioritario dentro de la cadena de valor para la obtención de un bien o servicio.
- Seguridad del paciente. Clasificación utilizada para indicadores que monitorean el uso de buenas prácticas preventivas para disminución de fallas, que son causas de eventos adversos, en el otorgamiento de atención al paciente ${ }^{9}$.

- Oferta. Son las propuestas de valor que se ofrecen al cliente o usuario, en sus productos o servicios.

- Calidad. Abarca la satisfacción del cliente o usuario con los bienes o servicios que recibe.

- Instancias regulatorias de salud ${ }^{6}$. Señalan y adicionan indicadores que designan como obligatorios entidades externas sanitarias y de seguimiento de observancia obligatoria en beneficio de la calidad y la seguridad ofrecidas al paciente.

6) KPI de mandos medios y alta dirección $n^{6}$. Los tableros estratégicos KPI son una herramienta diseñada en una estructura matricial. En la parte lateral de la matriz se establecen las diversas jerarquizaciones de categorización, denominadas perspectivas y seleccionadas por los actores de los procesos. En la parte superior derecha se incluyen los datos relevantes del KPI que se pretende visualizar después de ser medidos. La descripción de su diseño estructural es:

- Perspectiva. Permite categorizar los indicadores: rentabilidad (productividad), actividades, seguridad del paciente, oferta, calidad e instancias regulatorias de salud.

- Áreas médicas. Especifica el área de la estructura orgánica del puesto.

- Objetivo. Identifica y expresa cualitativamente las finalidades hacia las cuales deben dirigirse los recursos, los esfuerzos y las actividades de la organización para dar cumplimiento a su misión y visión.

- Estrategia. Iniciativa que establece cómo se alcanzarán el objetivo y la meta que se ambicionan para obtener los resultados esperados.

- Indicador. Medio, vínculo o variable para medir valores en una escala de medición, derivados de una serie de hechos observados en las actividades de prioridad de los procesos.

- Resultados del indicador. Cobran sentido de acuerdo con los objetivos establecidos (resultados esperados) a partir de los cuales se organiza una serie de actividades para alcanzarlos. Incluye: 
- Meta. Es un elemento cuantitativo del objetivo en un tiempo.

- Estatus. Es la parametrización de acuerdo con el avance de los resultados y se le asigna color verde, rojo o amarillo dependiendo del límite máximo o mínimo permisible.

- Gráfico. Ilustra las tendencias del KPI de forma visual para analizar, comprender y hacer comparaciones, entre otros beneficios.

\section{Categorización de indicadores}

Otro aspecto de análisis es la clasificación de indicadores, en primer lugar por el ámbito de medición²:

- Indicadores de insumos (inputs): cuantifican los recursos físicos, humanos, financieros, tecnológicos y de infraestructura, entre otros; son requerimientos en la producción de los bienes y servicios para el logro final de los resultados.

- Indicadores de procesos: aquellos KPI que miden el desempeño de las actividades vinculadas con la ejecución o forma en que el trabajo es realizado para producir los bienes y servicios.

- Indicadores de productos: muestra los KPI en los bienes y servicios de manera cuantitativa producidos por una organización.

- Indicadores de resultado final o impacto: KPI que miden los resultados en cuanto al fin último esperado con la entrega de los bienes y servicios.

La segunda categorización se refiere a los objetivos relacionados con los procesos que se encuentren en el nivel de actividades, los concernientes a los bienes y servicios, a componentes de calidad y los de resultados e impacto en el nivel de propósito y fin, respectivamente. Los indicadores de impacto permiten verificar los fines sociales o económicos a los que contribuye el programa social ${ }^{10}$.

La tercera clasificación se presenta a continuación con los indicadores con alineamiento estratégico, táctico y operativo, que permiten coherencia y claridad en la estructura formal de la organización ${ }^{11}$ :

- Indicadores estratégicos. KPI que se establecen para evaluar; son parámetros de medición del cumplimento de la implementación de la estrategia para sus objetivos y el alcance de resultados esperados y su impacto. Están ubicados en el nivel corporativo, la alta dirección de la organización.

- Indicadores tácticos. KPI que corresponden a los parámetros de medición del cumplimento de la ejecución del programa de trabajo o los proyectos para el alcance de sus objetivos específicos y la obtención de los resultados esperados. Están ubicados en los departamentos que se conoce como mandos medios.

- Indicadores de operación. En el último nivel, pero no menos importante, están los KPI con parámetros de medición del cumplimento de la ejecución de actividades del proceso o proyectos para el logro de sus objetivos particulares, y el alcance de los resultados esperados en la producción del bien o servicio. Están ubicados en la operación, y finalmente son los dueños y actores del proceso.

\section{Correlación de los indicadores de desempeño con alineamiento estratégico y los procesos médicos}

En la figura 1 se analizan y correlacionan los KPI en alineamiento estratégico en una homogenización de criterios con los procesos. Se observa y se complementa la necesidad de establecer de manera cotidiana indicadores no solo en resultados, sino desde la entrada, durante las actividades de prioridad del proceso y en los resultados, además de correlacionar o asociar los niveles estratégicos con los procesos de las organizaciones, con la finalidad de ser más preventivos en la medición y ser correctivos en las acciones de mejora, e inclusive de impacto hacia el exterior del proceso en entidades externas en las cuales repercute. Por ello, para definir un indicador se deben considerar sus relaciones con el modelo de planeación estratégica ${ }^{2,11,12}$.

Por lo tanto, en la actualidad, los KPI tienen una participación importante en la competitividad, lo cual se basa en contar con un plan que contemple misión, visión, objetivos y estrategias corporativas. Además, es necesario incluir los KPI en las entradas de insumos, en las actividades de mayor importancia y en la salida del proceso:

- Los key process input variable (KPIV) o indicadores clave de desempeño de entradas de insumos o requerimientos. Se consideran los KPI correspondientes a los insumos de los recursos tanto físicos como humanos, financieros, tecnológicos y de infraestructura, entre otros; cuantificados en requerimientos en la producción de bienes y servicios para el logro de resultados esperados de productos o servicios.

- Los key process variable (KPV) o indicadores clave de desempeño de los procesos o de las actividades de transformación de prioridad o claves. Se 


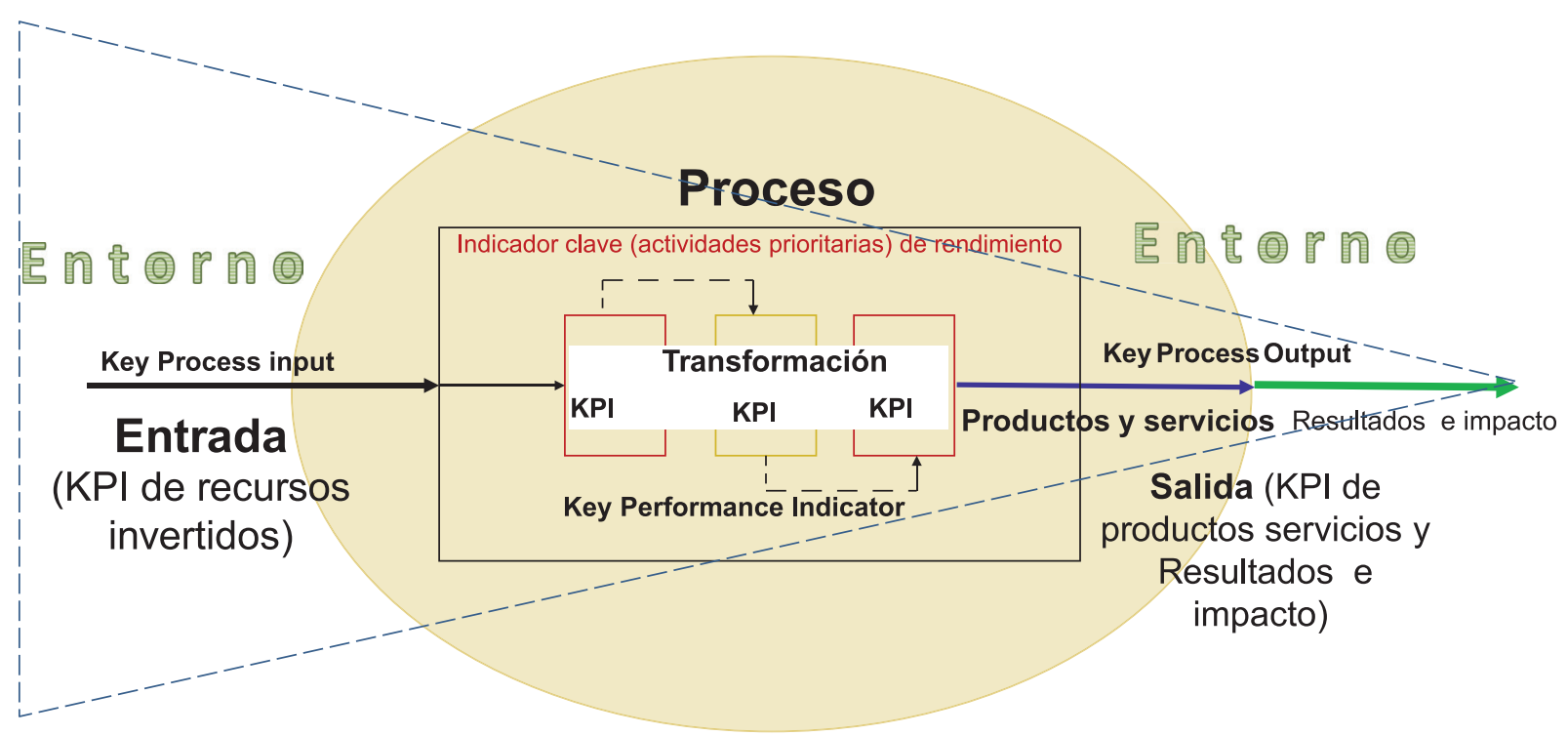

Figura 1. Indicadores según el ámbito de medición.

consideran los indicadores dentro de la producción de bienes o servicios, o bien en la cadena de producción e inclusive en la cadena logística y de suministro de las actividades primarias. Sin embargo, se define "proceso", tema fundamental de este estudio, como bien señala el Instituto Nacional de Estadística y Geografía (2002), como «una serie de actividades lógicas y ordenadas que deben ser definibles, repetibles, predecibles, continúas o cíclicas, mensurables, que llevan a la obtención de un producto que puede ser un bien o un servicio»; por ejemplo, los procesos de hospitalización, urgencias, consulta externa y cirugía, entre otros

- Los key process output variable (KPVO) o indicadores clave de desempeño de salidas, resultados, productos o servicios, e inclusive de impacto. Se consideran los KPI correspondientes a medir la satisfacción de los resultados en cuanto al fin último esperado (impacto) con la entrega de los bienes y servicios.

\section{Correlación de los indicadores de desempeño de procesos médicos con alineamiento estratégico}

Por todo lo anterior se hace necesario y se da importancia al entendimiento de la estandarización de los procesos médicos y de apoyo con el propósito de mejorarlos en sus diversas actividades de prioridad correlacionadas y alineadas con la estrategia de la organización. En la figura 2 se muestra cómo se complementa la necesidad de establecer de manera

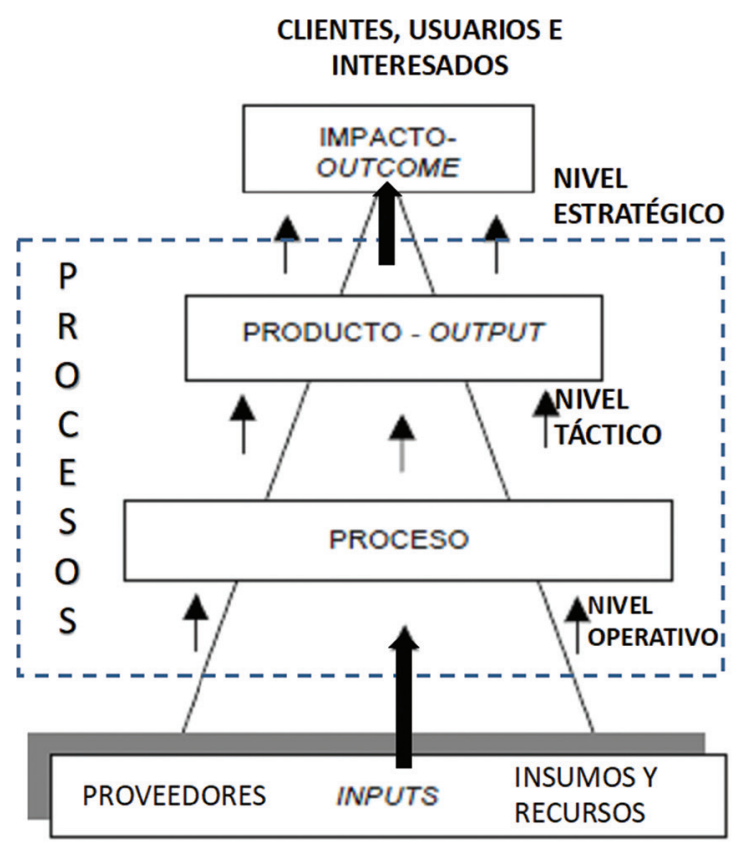

Figura 2. Modelo de KPI para actividades de prioridad del proceso y niveles de alineamiento estratégico.

cotidiana indicadores no solo en resultados, sino desde la entrada, durante las actividades de prioridad dentro del proceso y en los resultados, con la finalidad de ser más preventivos en la medición y ser correctivos en las acciones de mejora. Por ello, para definir un indicador se debe considerar la relación del proceso con el modelo de planeación estratégica ${ }^{13}$.

En la figura 2 se pueden distinguir las actividades de prioridad que se identifican en la cadena de valor como actividades únicas y distintivas que entregan de forma 


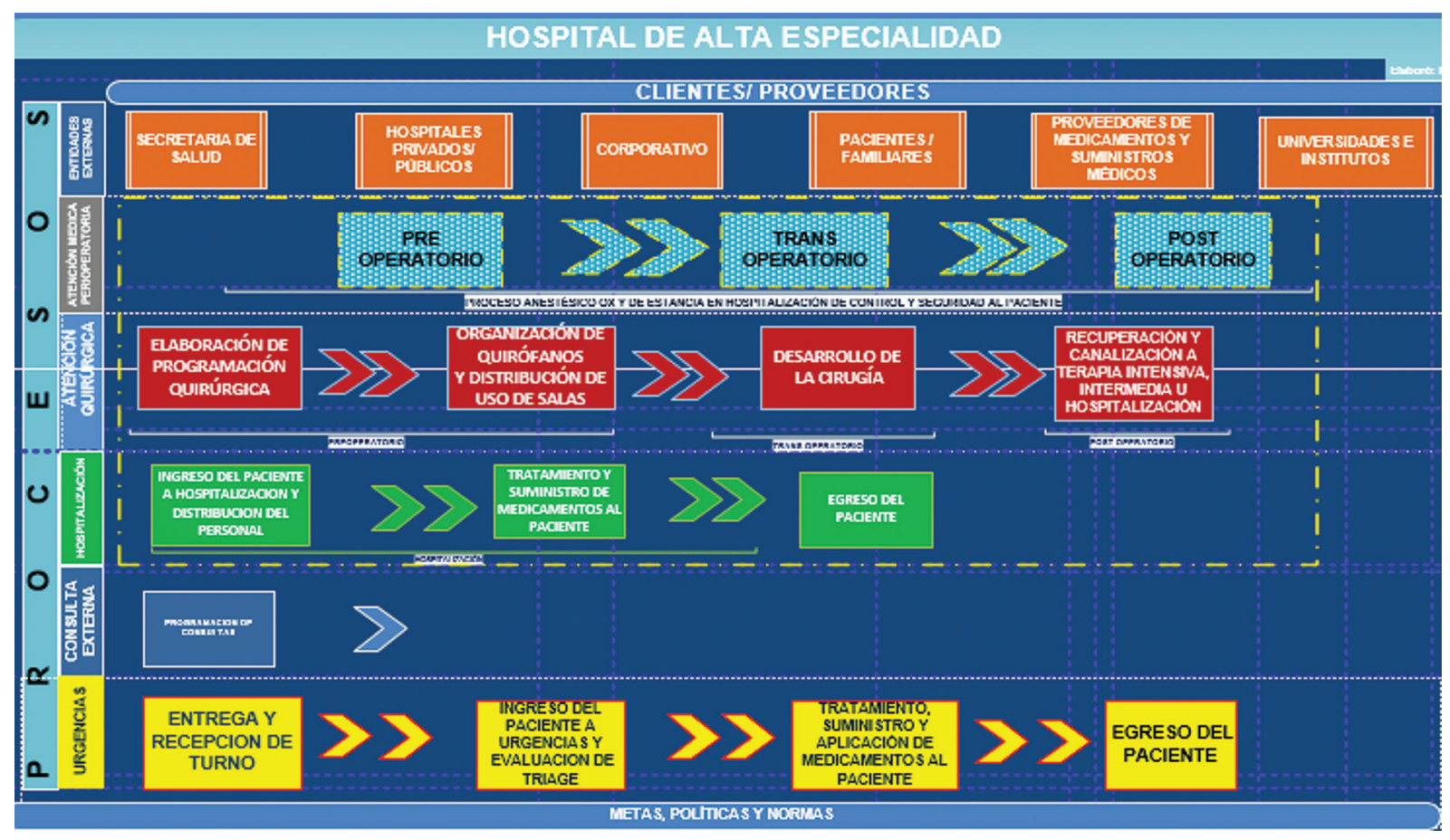

Figura 3. Modelo de macroprocesos de un hospital de alta especialidad. Fuente: elaboración propia.

EFICACIA DE CIRUGIA $50.72 \%$

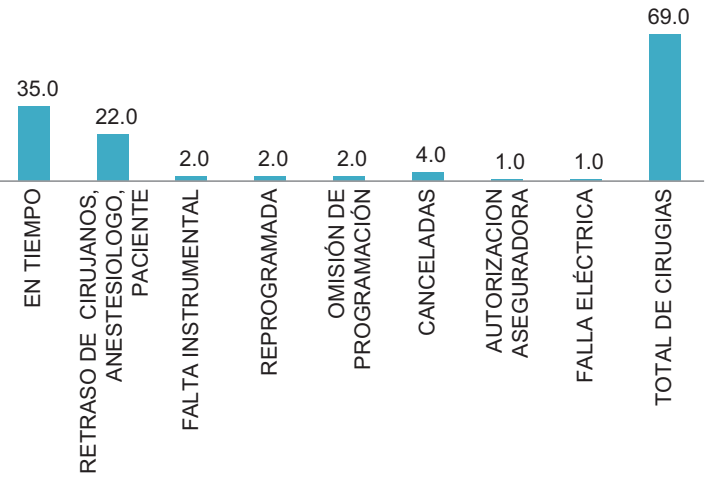

Figura 4. Eficacia de cirugías en tiempo programado contra lo que realmente se realizó con oportunidad.

satisfactoria el bien o servicio del proceso. Además, se sobrepone un punteado triangular que representa la visualización y desdoble de la pirámide estratégica que deberá implementar la organización, institución o empresa en sus niveles operativo, táctico y estratégico, priorizando indicadores de acuerdo con el nivel jerárquico involucrado dentro el proceso, no solo el productivo, sustantivo, principal, sino en todos los procesos de soporte de la organización en sus diversas áreas, como finanzas, mercadotecnia, recursos humanos, investigación y desarrollo, sistemas de información, etcétera ${ }^{11-13}$.
A modo de ejemplo, en la figura 3 se muestra la arquitectura del macroproceso de un hospital de alta especialidad $^{6,13}$. Se refiere a procesos generales del hospital, se trabaja en paralelo al desarrollo de esta serie de entrevistas como integración general de sus procesos, cuyas ventajas permiten ver el inicio y el fin del proceso, se trasladó a estructuras planas y horizontales, no aparecen los nombres o puestos de los responsables de la estructura orgánica, lo cual permite un diagnóstico que no crea conflictos entre las personas y visualiza que son un equipo de desempeño que realiza las actividades y que es necesario medir antes y durante el proceso para entregar resultados esperados por la alta dirección.

En la tabla 1, para seguir el ejemplo, se muestran la construcción y el tablero de indicadores para observar su aplicación en cirugía ${ }^{6,13}$, y se observan la estrategia como medio para alcanzar el objetivo y el indicador de eficacia quirúrgica programada en relación con las cirugías realizadas, y que afecta la calidad y seguridad para el paciente (Fig. 4).

\section{Discusión}

Para construir indicadores y visualizar su formalización para implementar e implantar el indicador es necesaria una ficha de trabajo, en la que además se 
Tabla 1. Construcción de tableros de indicadores para su aplicación en cirugía

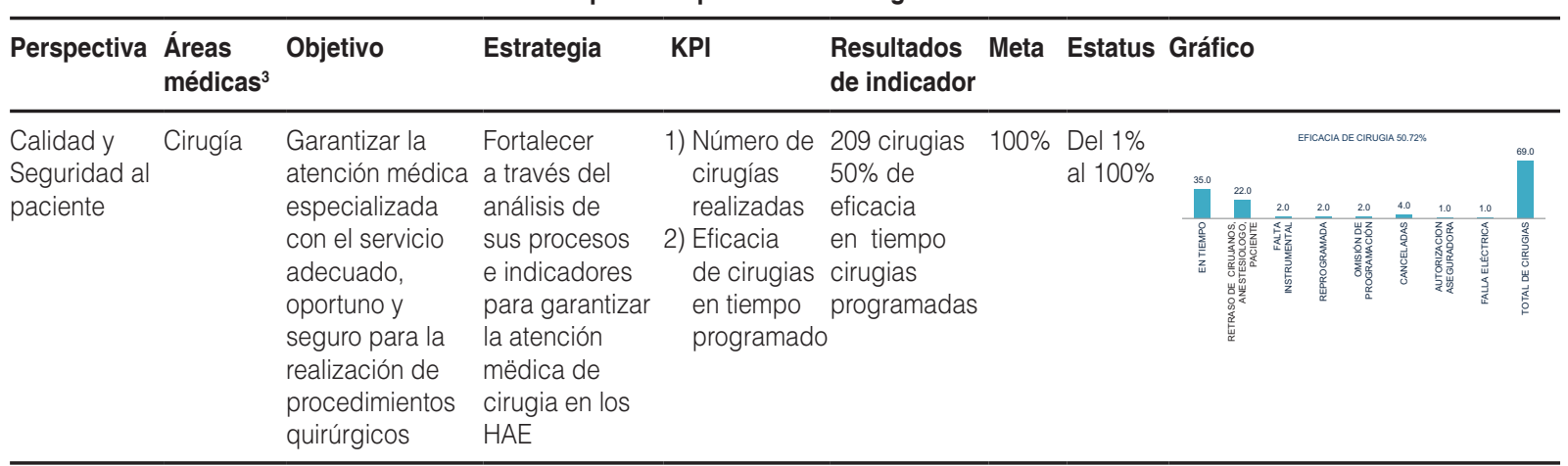

deberá detallar quién o quiénes seran los responsables de su construcción y seguimiento, así como de mantener actualizado el indicador e incluir en su formato al menos los siguientes elementos e información:

- Encabezado. Nombre de la organización y área correspondiende al nivel de la estructura orgánica.

- Tener el nombre de: Ficha de indicador.

- Detallar si es indicador de estrategia, objetivo, proyecto o proceso.

- Nombre del indicador.

- Definición del indicador. Se describe el indicador de acuerdo con los conceptos o variables que lo componen. Debe incluir los aspectos escenciales de manera sintética.

- Algoritmo. Expresa algebraicamente cómo se obtiene el indicador. Debe incluir la unidad de medida de cada componente y la fórmula si es necesario.

- Objetivo del indicador. En todos los casos es medir.

- Fuentes de datos. Conjunto de características técnicas sobre las fuentes de información de los datos que componen el indicador:

a)Fuente: nombre de la unidad, el establecimiento o el organismo que elabora la información.

b) Cobertura geográfica: expresa la delimitación territorial a la que corresponde la información. Puede ser nacional, por sectores, por áreas, etcétera.

c) Unidad de observación: define las unidades que la fuente de información tiene para la obtención de datos; por ejemplo, eventos, personas, encuesta, fallas, quejas, errores, inconsistencias, entre otros.
d)Fecha de disponibilidad de la información: día en que se da a conocer la información.

- Construcción del indicador:

a) Responsable de construir el indicador. Nombre de la persona responsable.

b) Fecha de actualización.

c) Observaciones. Se presentarán elementos que permitan la mayor claridad sobre el indicador y referencias metodológicas estadísticas, como muestra de la población.

d) Gráfico. Se presenta de forma visual y estadística la construcción de datos y resultados.

En conclusión, se define que un indicador es la representación numérica de una variable que revela cambios en función del tiempo. Es la expresión, por lo general numérica, del análisis del proceso identificado, y su medición es a través de un algoritmo o fórmula, que facilita el acceso a la información a diferentes usuarios para transformar la información en iniciativas estratégicas y acciones de mejora.

Es relevante puntualizar algunos apectos importantes, destacar y tener presente:

- Que en todos los casos el objetivo de un indicador es medir el cumplimiento, el objetivo, la ejecución del proceso, proyecto o la implementación de la estrategia.

- Que los indicadores, además de medir y permanecer en un constante monitoreo para el alcance de resultados, es conveniente y muy útil establecerlos para medir durante el proceso, lo cual permitirá garantizar y entender esta palabra como prevención, para obtener resultados esperados, con el propósito de satisfacer las expectativas de los diversos actores del proceso en su cadena de valor, donde se suministren e ingresen los requerimientos de calidad, se atiendan las actividades de operación y se prioricen para 
entonces para monitorear con KPI, para dar la respuesta en resultados esperados, que pueden convertirse dependiendo de la organización en bienes o servicios que satisfagan al cliente, usuario o interesado.

Además, se debe tener alineamiento estratégico en sus niveles organizativos o jerárquicos correspondientes, con el establecimiento y la implementación de acuerdo con las prioridades de sus actividades con indicadores que permitan garantizar el alineamiento hacia la misión, visión de objetivos estratégicos y en su caso implantar las líneas de acción o las iniciativas correspondientes en tiempo que permitan redireccionar o reivindicar acciones estratégicas hacia los resultados esperados en la producción de bienes y servicios.

En definitiva, los indicadores hay que documentarlos y formalizarlos para que se dé seguimiento en su monitoreo y continuidad entre una administración y otra, a través de fichas de indicadores, para que posteriormente se clasifiquen de acuerdo con las perspectivas de mayor conveniencia en la organización, con alineamiento coherente y que tras hacer pruebas piloto se incorporen a la automatización correspondiente.

\section{Agradecimientos}

Gracias a la Dirección General de Asuntos del Personal Académico, de la Universidad Nacional Autónoma de México, por el apoyo otorgado en la estancia posdoctoral.

\section{Responsabilidades éticas}

Protección de personas y animales. Los autores declaran que para esta investigación no se han realizado experimentos en seres humanos ni en animales.
Confidencialidad de los datos. Los autores declaran que han seguido los protocolos de su centro de trabajo sobre la publicación de datos de pacientes.

Derecho a la privacidad y consentimiento informado. Los autores declaran que en este artículo no aparecen datos de pacientes.

\section{Conflicto de intereses}

La autora declara no tener ningún conflicto de intereses.

\section{Bibliografía}

1. Rodríguez I, Alpuin D. La gestión por procesos en las organizaciones. La forma en la que los resultados se logran. Reino Unido: Deloitte Touche Tohmatsu Ltd; 2014. Disponible en: www.deloitte.com/about.

2. Armijo M, Bonnefoy J. Indicadores de desempeño del sector público. Organización de las Naciones Unidas; 2005.

3. Hernández Rodríguez S. Administración. Teoría, proceso, áras funcionales y estrategias para la competitividad. 2. ${ }^{a}$ ed. México: McGraw-Hill Interamericana; 2008.

4. INEGI. Guía para la elaboración del Programa Institucional de DesarroIlo Informático. Instituto Nacional de Estadística y Geografia; 2002.

5. Beltrán Sanz J, Carmona Calvo MA, Carrasco Pérez R, Rivas Zapata MA, Tejedor Panchon F. Guía para una gestión basada en procesos. Instituto Andaluz de Tecnología; 2002.

6. Torres-Salgado MK, Vega-Lebrún CA. Arquitectura de indicadores estratégicos para elevar la calidad de los servicios médicos de los hospitales de alta especialidad. Investigación y Ciencia de La Universidad Autónoma de Aguascalientes. 2018;73:84-94.

7. Porter ME. "What is Strategy". Harvard Business Review. 1996;(60-78). Reprint R0902H 2011.

8. Simon D, Fischbach K, Schoder D. Enterprise architecture management and its role in corporate strategic management. Information Systems and e-Business Management. 2013;12:5-42.

9. Meena K, Thakkar J. Development of balanced scorecard for healthcare using interpretive structural modeling and analytic network orocess. J Adv Manag Res. 2014;11:232-56.

10. Consejo Nacional de Evaluación. Manual para el diseño y la construcción de indicadores. Instrumentos principales para el monitoreo. Consejo Nacional de Evaluación de la Política de Desarrollo Social. México: CONEVAL; 2019.

11. David FR. Conceptos de administración estratégica. 9. ${ }^{\text {a ed. Naucalpan }}$ de Juárez: Pearson Educación de México, S.A. de C.V.; 2013. Disponible en: https://maliaoceano.files.wordpress.com/2017/03/libro-fred-david-9a-edicion-con-estrategica-fred-david.pdf.

12. Ludwing von Bertalanffy K. Teoría general de sistemas. México: Fondo de cultura Económica; 2004.

13. Torres-Salgado MK. Arquitectura de procesos con indicadores estratégicos en los servicios médicos de consulta externa en hospitales de alta especialidad. Gac Med Mex. 2019;155:576-84. 